(T1), follow-up (T2 15 days after) and post-test (T3 30 days after).

Results A total of 56 patients were enrolled in the study. They were randomized assigned to either the intervention group $[\mathrm{IG}](\mathrm{n}=28)$ or to control group [CG] $(\mathrm{n}=28)$. Over time the hope indexes in the IG compared to the CG, suggest a positive effect of HPP in the increase of hope over time. The most expressive effect was observed in the total hope level and in the gains in positive interior disposition and expectations, with significant differences in each of the data collection points in time.

Conclusions Further research should create training programs for nurses and nursing students within the scope of the promotion of hope, in order to promote the development of personal and professional skills.

\section{WHAT ARE THE PALLIATIVE CARE NEEDS OF PATIENTS UNDERGOING CHIMERIC ANTIGEN RECEPTOR (CAR) T- CELL THERAPY FOR REFRACTORY DIFFUSE LARGE B- CELL LYMPHOMA/PRIMARY MEDIASTINAL B-CELL LYMPHOMA AT A REGIONAL CENTRE?}

Charlotte Stenson, Jennifer Vidrine, Felicity Dewhurst. Newcastle-Upon-Tyne Hospitals NHS Foundation Trust

\subsection{6/spcare-2021-PCC.140}

Background Evidence suggests palliative care is often initiated late in patients with haematological malignancy; this is complicated by the challenging dichotomy of advanced disease and treatment with curative intent. Chimeric antigen receptor (CAR) T-cell therapy has recently been approved by NICE in some UK centres for diffuse large B-cell lymphoma (DLBCL) and primary mediastinal B-cell lymphoma (PMBCL) after failure of two lines of treatment. Outcomes of this group are poor and treatment may be the last hope for remission or cure. CAR T-cells are associated with unique toxicities, sometimes requiring aggressive supportive care. Little is known about the palliative care needs of these patients.

Methods Detailed quantitative and qualitative retrospective case note analysis of all patients receiving CAR T-cell infusion between January 2019 and June 2020 at the Northern Centre for Cancer Care, Newcastle-Upon-Tyne.

Results 33 patients received CAR T-cell therapy. Overall, symptom burden was significant. Four core symptoms were assessed: nausea/vomiting, pain, dyspnoea, and anxiety/psychological distress. All patients experienced at least one symptom, and $82 \%$ of patients experienced two or more symptoms.

6 month mortality was high (51\%). A third of patients were admitted to Intensive Care due to treatment toxicity and this group had a higher mortality (64\%). 8 patients were referred to the palliative care service during admission. Of the 8 patients seen by palliative care, 7 had evidence of advance care planning (ACP). There was no evidence of ACP for the patients who died without palliative care input.

Conclusions Patients undergoing CAR T-cell therapy for DLBCL/PMBCL have evidence of unmet palliative care needs with significant symptom burden and high mortality. The added challenge of many patients travelling from outside the region for treatment may be a barrier to initiation of ACP. More work is needed on the impact of integrated, upstream palliative care in this group.

\section{3 IMPROVING QUALITY OF LIFE IN MALIGNANT ASCITES WITH INDWELLING PERITONEAL DRAINS}

Joanna Coleman, Leonie Armstrong, Jennifer Grieve, Jeanette Doran, Katie Frew, Sarah Robinson, Robert Johnston, Karl Jackson, Avinash Aujayeb. Northumbria HealtCare NHS Foundation Trust

\subsection{6/spcare-2021-PCC.141}

Introduction Traditional management of malignancy related ascites (MRA) is via large volume paracentesis (LVP). NICE recommends indwelling peritoneal catheters, (IPCs) which are cost effective and patient centred. A service and practice guidelines were set up. Ongoing reviews assess safety and efficacy and inform future pathways.

Methods A single centre retrospective analysis of patients requiring IPCs between October 2018 and August 2020 was performed. Demographics and outcomes were collected. Descriptive statistical methodology was applied. IPCs are placed in theatre or dedicated clean spaces, with pre-operative antibiotics.

Results 27 patients (14 male, 13 female) underwent 28 IPC placements. [1 patient had 2 IPCs (this will not be discussed as case is published)]. Diagnoses were gastrointestinal (13), breast (2), ovarian (5), prostate (1), thymic (1), unknown primary (4) cancers and 1 mesothelioma. 3 had no preceding LVP; 24 had a mean of 2.2 LVPs before. 6 developed postoperative leaks. 2 patients developed cellulitis. Bacterial colonisation occurred in 1 patient. 4 IPCs were removed: 2 as ascites resolved, 1 for tumour infiltration, 1 for non-resolving site cellulitis. 24 patients have died: mean number of days to death: 56.4 (range 6-262). Integrated Palliative Care Outcome Scale scores collected in 20 patients consistently show sustained reduction in pain, dyspnoea, nausea, vomiting, drowsiness, distension and improvements in body image and appetite.

Conclusions IPCs are safe and efficient. More formal evidence is required on some aspects. A grant application has been submitted to the Royal College of Physicians for the Dorothy Whitney-Wood scholarship to perform a systematic review of the literature, a survey through the APM and an open labelled prospective study to determine if patients with MRA should have an IPC at first presentation and correlate with quality of life outcomes. We also propose sequential analysis of peritoneal fluid to assess bacterial colonisation and effect on outcomes.

\section{ULTRASOUND USE IN THE COMMUNITY SETTING}

Leena Patel, Lara Wood, Kirsty Cowper, Isobel Morton. St Joseph's Hospice

10.1136/spcare-2021-PCC. 142

Background Ultrasound is an increasingly important diagnostic and therapeutic tool in palliative care. Ultrasound use within the community, prevents hospital admissions and supports preferred places of care. Few studies describe the indications, outcomes and complications of ultrasound use in a community setting.

Aim Review and evaluate the use of Focused Abdominal Ultrasound (FASP) at St Joseph's Hospice, over six years.

Methods A retrospective review of data from ultrasound logbooks and computerised clinical records between May 2012 April 2020. 
Results 410 ultrasounds were performed, $3(<1 \%)$ of these were in the patient's own home. All scans were performed by a Palliative Medicine Consultant or Clinical Fellow trained in use of FASP.

Clinical indication was broadly split into malignant and non-malignant, $64 \%$ vs.36\% respectively.

Identified indications include; ascites 74\% (302); urinary retention 19\% (78); constipation or overflow 5\% (9); deep vein thrombosis $1 \%$ (5); pleural effusion <1\% (3); assessment of mass $<1 \%$ (2) and spontaneous bacterial peritonitis $<1 \%$ (1). $41 \%$ of the 302 ultrasounds for ascites, resulted in paracentesis. Major and minor complications rates, $0 \%$ vs. $8 \%$ respectively, were reviewed over 4 weeks. Failure of procedure was the highest minor complication rate at $2 \%$. Persistent leakage from drain site, infection, local skin reaction and an asymptomatic drop in blood pressure had complication rates of $<1 \%$ each. Data trends show the number of ultrasounds performed each year is decreasing, however, paracentesis rates remain relatively stable, suggesting more targeted use.

Conclusions Community ultrasounds reduce hospital transfers, admissions and need to access hospital-based radiology services. With appropriately selected patients, the low complication rates, support continued use of community paracentesis. Patient views and preferences on the location of performing ultrasounds and procedures would be valuable. This combined with projected cost savings, has the potential to demonstrate and endorse the importance of these community-based services.

\section{A REVIEW OF PATIENTS REQUIRING LARGE VOLUME PARACENTESIS FOR MALIGNANT ASCITES}

Sadé Hacking, Katie Frew, Irfan Iqbal Khan, Muhammad Tahir Chohan, Avinash Aujayeb. Northumbria HealtCare NHS Foundation Trust

\subsection{6/spcare-2021-PCC.143}

Introduction Malignancy-related ascites (MRA) carries significant morbidity and mortality, and is usually treated by large volume paracentesis (LVP). There are no local guidelines. LVP occurs via Medical Ambulatory Care (MAC). An indwelling peritoneal catheter (IPC) service exists for recurrent ascites. We hypothesised that analysis of patients with MRA requiring LVP would allow protocol development.

Methods A retrospective analysis of patients requiring LVPs for MRA on MAC between Oct 2018 and Dec 19 was performed with local Caldicott approval. Demographics and outcomes were collected. Descriptive statistical methodology was applied with continuous data presented as mean (standard deviation (SD); range) and categorical variables as frequencies or percentages.

Results 27 patients were identified \{Mean age 68.5 yrs (SD \pm 11.85 ; range: $41-86$ ); $55.5 \%$ female $\}$. Rockwood Clinical Frailty Score was recorded in 18: $1=7.4 \%, 2=18.5 \%$, $3=14.8 \%, 5=7.4 \%$. All patients had at least 2 admissions in the preceding 12 months (mean 5.5 (SD $\pm 2.76 ; 2-15)$. Diagnoses were GI (55.5\%), ovarian (25.9\%), unknown primary $(11.1 \%)$, breast $(3.7 \%)$, and neuroendocrine cancers $(3.7 \%)$. 92\% had metastatic disease, 55\% peritoneal metastases. Presenting symptoms were abdominal swelling/bloating/distension $(100 \%)$, abdominal pain $(22 \%)$ and shortness of breath (11\%). $40.7 \%$ of were readmitted within 30 days for drainage. $37 \%$ were referred and had an IPC. Mean time from first LVP to death was 58.18 days (SD $\pm 54.00 ; 7-210$ ). $66 \%$ of patients had a sample sent for analysis. $38.88 \%$ had positive cytology. 5 samples had biochemical analysis: 4 were transudative (all negative cytology); 1 was exudative (positive cytology). No patients developed hypotension with LVP. 1 patient with transudative MRA developed bacterial peritonitis.

Conclusions This is the first study to determine epidemiological local data on the foregoing. There is clear variance in practice. We suggest that all samples should have biochemical analysis (transudates are to be given prophylactic antibiotics) and all patients should be referred for consideration of IPC.

\section{Posters 126 - 138 | Symptom Management}

\section{A SCOPING REVIEW OF THE IMPLEMENTATION OF NON-MEDICAL DEVICES FOR THE SELF-MANAGEMENT OF CHRONIC BREATHLESSNESS}

Aliya Syahreni Prihartadi, Giovanna Impelliziere Licastro, Mark Pearson, Miriam J Johnson, Tim Luckett, Flavia Swan. Wolfson Palliative Care Research Centre, Hull York Medical School, University of Hull, Kingston-Upon-Hull, Faculty of Health, University of Technology Sydney (UTS), Sydney, Australia

\subsection{6/spcare-2021-PCC.144}

Background Non-medical devices such as the handheld fan (fan), mobility aids and inspiratory muscle training (IMT) devices offer important benefits for patient self-management of chronic breathlessness. We examined the published evidence regarding barriers and facilitators to implementation of nonmedical devices for the self-management of chronic breathlessness from the perspectives of patients, carers and clinicians.

Methods MEDLINE, EMBASE, SCOPUS, EBSCO and the Cochrane Database of Systematic Reviews were searched. Papers were imported into EndNote and Rayyan for review against a priori eligibility criteria. These were; i) any study design including randomised controlled trials, observational, interviews, primary and secondary analyses of data, ii) patients; adults with chronic breathlessness, carers', and clinicians, iii) exposure to the fan, mobility aids and IMT devices, iv) and outcome data regarding implementation of these devices for self-management of chronic breathlessness.

Data relevant to use were extracted and categorised as potential implementation barriers and facilitators, and a narrative synthesis exploring reasons for similarities and differences conducted.

Results Seven studies were included; five fan and two mobility aid (wheeled walkers with seats) studies. All of the studies presented patient use of non-medical devices only. The fan was implemented for self-management of chronic breathlessness at home whereas mobility aids were used mainly for outdoor activities. Key barriers to implementation of non-medical devices were: appearance; credibility; self-stigma; technical specifications. Common facilitators were ease of use and feeling safe and secure with the device.

Conclusion Limited evidence exists for the implementation of non-medical devices by patients for the self-management of chronic breathlessness. We highlight the lack of studies on carers, clinicians, IMT devices and the use of applied implementation science theory. Further implementation research is 